\title{
The Dynamical Behaviors for a Class of Immunogenic Tumor Model with Delay
}

\author{
Ping Bi, ${ }^{1}$ Zijian Liu, ${ }^{2}$ Mutei Damaris Muthoni, ${ }^{1}$ and Jianhua Pang ${ }^{3}$ \\ ${ }^{1}$ Department of Mathematics, Shanghai Key Laboratory of PMMP, East China Normal University, 500 Dongchuan Rd., \\ Shanghai 200241, China \\ ${ }^{2}$ College of Mathematics and Statistics, Chongqing Jiaotong University, Chongqing 400074, China \\ ${ }^{3}$ School of Science, Guangxi University of Science and Technology, Liuzhou 545006, China
}

Correspondence should be addressed to Ping Bi; pbi@math.ecnu.edu.cn

Received 18 May 2017; Revised 13 July 2017; Accepted 9 August 2017; Published 25 October 2017

Academic Editor: Po-Hsiang Tsui

Copyright (C) 2017 Ping Bi et al. This is an open access article distributed under the Creative Commons Attribution License, which permits unrestricted use, distribution, and reproduction in any medium, provided the original work is properly cited.

This paper aims at studying the model proposed by Kuznetsov and Taylor in 1994. Inspired by Mayer et al., time delay is introduced in the general model. The dynamic behaviors of this model are studied, which include the existence and stability of the equilibria and Hopf bifurcation of the model with discrete delays. The properties of the bifurcated periodic solutions are studied by using the normal form on the center manifold. Numerical examples and simulations are given to illustrate the bifurcation analysis and the obtained results.

\section{Introduction}

For the longest time, malignant tumors have posed a threat to human lives. Effective strategies based on the immune system have been championed to complement traditional methods of cancer treatment, such as surgery, radiation, and chemotherapy. Cancer immunotherapy is the use of the immune system to treat cancer. Immunotherapy is used to provoke the immune system into attacking the tumor cells by using these cancer antigens as targets. Cell types that can be used in this way are natural killer cells, lymphokine-activated killer cells, cytotoxic $\mathrm{T}$ cells, and dendritic cells. There has been much interest in mathematical models describing the interaction between tumor cells and effector cells of the immune system; see [1-6]. An ideal model can provide insight into the dynamics of interactions of the immune response with the tumor and may play an important role in understanding of cancer and developing effective drug therapies. Developing ideal models for such complex processes is not easy. Simple models which display some useful immunological phenomena have been proposed and studied extensively. See Bi and Xiao [7], Galach [4], and Yafia [8-10], and the references cited therein. In 1994, Kuznetsov et al. [11] introduced a model, which describes competition between the tumor and immune cells. It also describes the response of effector cells (ECs) to the growth of tumor cells (TCs). It is assumed that the tumor cells are "immunogenic" and thus subject to immune attack by cytotoxic (CT) or natural killer (NK) cells. This model studies the infiltration of TCs by ECs and also the possibility of ECs inactivation. It is assumed that interactions between ECs and TCs in vitro can be described by the following kinetic scheme describing interactions between ECs and TCs:

$$
E+T \underset{k_{-1}}{\stackrel{k_{1}}{\rightleftarrows}} C\left\{\begin{array}{l}
\stackrel{k_{2}}{\longrightarrow} E^{*}+T \\
\underset{k_{3}}{\longrightarrow} T^{*}+E
\end{array}\right.
$$

where $E, T, C, T^{*}, E^{*}$ are the local concentrations of $E C s$, TCs, EC-TC complexes, inactivated ECs, and TCs, respectively. The parameters $k_{1}, k_{-1}, k_{2}$ and $k_{3}$ are nonnegative constants, which describe the conversion rates of differential cells. Then Kuznetsov and Taylor's model is as follows:

$$
\begin{aligned}
& \frac{d E}{d t}=s+F(C, T)-d_{1} E-k_{1} E T+\left(k_{-1}+k_{3}\right) C, \\
& \frac{d T}{d t}=a T\left(1-b T_{\text {tot }}\right)-k_{1} E T+\left(k_{-1}+k_{2}\right) C,
\end{aligned}
$$




$$
\begin{aligned}
\frac{d C}{d t} & =k_{1} E T-\left(k_{-1}+k_{2}+k_{3}\right) C, \\
\frac{d T^{*}}{d t} & =k_{3} C-d_{2} T^{*}, \\
\frac{d E^{*}}{d t} & =k_{2} C-d_{3} E^{*},
\end{aligned}
$$

where $C$ is the normal rate of the flow of adult ECs into the tumor site, $F(C, T)$ describes the accumulation of effector cells in the tumor cells localization due to the presence of the tumor. $F(C, T)=f C /(g+T),(f, g$ are constants $)$. If $T_{\text {tot }} \approx$ $T\left(T_{\text {tot }}=T+C\right)$, it is reasonable to assume $d C / d t \approx 0$, that is, $C \approx k E T\left(k=k_{1} /\left(k_{-1}+k_{2}+k_{3}\right)\right)$, then $F(C, T)=F(E, T)$. Then we only need to analyze the dynamical behavior of the first two equations.

In 2003, Galach [4] suggested that the function $F$ is in the Michaelis-Menten form $F(E, T)=\theta E T$, and thus (2) can be simplified as

$$
\begin{aligned}
& \frac{d x}{d t}=c+\theta x y-m_{1} x y-d_{1} x, \\
& \frac{d y}{d t}=a y(1-b y)-m_{2} x y
\end{aligned}
$$

where $x$ is the local concentrations of ECs, and $y$ is the local concentrations of TCs, $m_{1}=k k_{2}, m_{2}=k k_{3}$, and all the parameters are positive. The properties of the model (3) is studied in [7-9]. The dynamical behaviors and the bifurcation of the model with delay are also studied by $[7,10]$.

In this paper, we analyze the dynamics of an immune response function with Michaelis-Menten form $F(E, T)=$ $p E T /(g+T)$, where $p$ and $g$ are positive constants, that is,

$$
\begin{aligned}
& \frac{d E}{d t}=s+\frac{p E T}{g+T}-m E T-d E, \\
& \frac{d T}{d t}=a T(1-b T)-n E T .
\end{aligned}
$$

In order to simplify the original model, we nondimensionalize (4) by choosing scale for $E$ and $T$ cell population, respectively. Let $\tau=n T_{0} t$, and replace $\tau$ with $t$; thus the model can be written as follows:

$$
\begin{aligned}
& \frac{d x}{d t}=\sigma+\frac{\alpha x y}{1+y}-\mu x y-\delta x, \\
& \frac{d y}{d t}=f y(1-\beta y)-x y,
\end{aligned}
$$

where $y=T / g, x=E / g, \tau=t n g, \sigma=s / n g^{2}, \alpha=$ $p / n g, \mu=m / n, \delta=d / n g, f=a / n g, \beta=b g$ and all the above parameters are positive.

This paper is organized as follows: In Section 2, the model without delay is considered, and the conditions for existence and stability of equilibria are given. In Section 3, the model with delay is studied. The existence of Hopf bifurcation is obtained; the direction and stability of bifurcated periodic solutions are also given with the help of center manifold and bifurcation theories. At the end of this paper, numerical results are given to illustrate the main results of this paper.

\section{Existence and Stability of the Equilibria}

It is easy to see that (5) has a tumor-free equilibrium $P_{0}=$ $(\sigma / \delta, 0)$. In order to find the positive equilibria $P_{1}=\left(x^{*}, y^{*}\right)$, we need to solve the following equations:

$$
\begin{aligned}
& \frac{d x}{d t}=\sigma+\frac{\alpha x y}{1+y}-\mu x y-\delta x=0, \\
& \frac{d y}{d t}=f y(1-\beta y)-x y=0 .
\end{aligned}
$$

Equation (6) is reduced to the following cubic polynomial:

$$
a_{1} y^{3}+a_{2} y^{2}+a_{3} y+a_{4}=0,
$$

where $a_{1}=f \beta \mu, a_{2}=f(-\alpha \beta+\beta \delta-\mu+\beta \mu), a_{3}=f \alpha-$ $f \delta+f \beta \delta-f \mu+\sigma, a_{4}=-f \delta+\sigma$. Obviously, system (5) in $R_{2}^{+}$ is as "well behaved" just as in biology. We have the following lemma by qualitative analysis.

Lemma 1. If $\mu>\alpha$, then the solutions of (5) are invariable in D:

$$
D=\left\{(x, y) \mid 0 \leq x \leq \frac{2 \sigma}{\delta}, 0 \leq y \leq \frac{2}{\beta}\right\} .
$$

Proof. If $x=0$ and $0<y<2 / \beta$, then

$$
\begin{aligned}
& \left.\frac{d x}{d t}\right|_{x=0}=\sigma>0, \\
& \left.\frac{d y}{d t}\right|_{x=0}=f y(1-\beta y) \begin{cases}>0 & \text { for } 0<y<\frac{1}{\beta}, \\
<0 & \text { for } \frac{1}{\beta}<y<\frac{2}{\beta} .\end{cases}
\end{aligned}
$$

Similarly, if $y=0$ and $0<x<2 \sigma / \delta$, one has

$$
\begin{aligned}
& \left.\frac{d x}{d t}\right|_{y=0}=\sigma-\delta x \begin{cases}>0, & \text { for } 0<x<\frac{\sigma}{\delta}, \\
<0, & \text { for } \frac{\sigma}{\delta}<x<\frac{2 \sigma}{\delta},\end{cases} \\
& \left.\frac{d y}{d t}\right|_{y=0}=0 .
\end{aligned}
$$

Also, if $y=2 / \beta$ and $0<x<2 \sigma / \delta$, we have

$$
\begin{aligned}
& \left.\frac{d y}{d t}\right|_{y=2 / \beta, 0<x<2 \sigma / \delta}=\left.y(f(1-\beta y)-x)\right|_{y=2 / \beta, 0<x<2 \sigma / \delta} \\
& \quad<0, \\
& \left.\frac{d x}{d t}\right|_{y=2 / \beta, 0<x<2 \sigma / \delta} \\
& \quad=\sigma+\left.x\left(\frac{2 \alpha \beta-(\beta+2)(2 \mu+\delta \beta)}{\beta(\beta+2)}\right)\right|_{y=2 / \beta, 0<x<2 \sigma / \delta} \\
& \quad<0,
\end{aligned}
$$


and, then, there exists a positive number $x^{*}$, which is given by

$$
x^{*}=\frac{\sigma \beta(\beta+2)}{(\beta+2)(2 \mu+\delta \beta)-2 \alpha \beta} .
$$

Such that $d x / d t<0$ when $x>x^{*}$. Similarly, $d x / d t>0$ when $x<x^{*}$. On the other hand, one has

$$
0<\frac{\sigma \beta(\beta+2)}{(\beta+2)(2 \mu+\delta \beta)-2 \alpha \beta}<\frac{2 \sigma}{\delta}
$$

with the help of $\mu>\alpha$. That is $0<x^{*}<2 \sigma / \delta$.

When $x=2 \sigma / \delta$ and $0<y<2 / \beta$, we can prove the result in a similar way. This finishes the proof.

It is easy to see that (5) has one tumor-free equilibrium $P_{0}=(\sigma / \delta, 0)$. With the distribution of the eigenvalues, we can easily obtain the following results.

Theorem 2. If $f>\sigma / \delta$, then $P_{0}$ is unstable. If $f=\sigma / \delta, P_{0}$ is stable. If $f<\sigma / \delta, P_{0}$ is asymptotically stable.

Theorem 3. If $>f \delta, \delta \beta>\mu>\alpha$, then $P_{0}$ is globally asymptotically stable.

Proof. When $>\delta f, \beta \delta>\mu>\alpha$, then it is easy to see that

$$
\begin{aligned}
& a_{1}>0, \\
& a_{4}=-f \delta+\sigma>0, \\
& a_{3}=f \alpha+(\sigma-f \delta)+f(\beta \delta-\mu)>0, \\
& a_{2}=f(\beta(\mu-\alpha)+(\beta \delta-\mu))>0 .
\end{aligned}
$$

This implies that $P_{0}$ is the only positive equilibrium.

Noting $\sigma>d f$, we know that $P_{0}$ is locally stable with the help of Theorem 2. In the remaining part of the proof, we only need to prove the global stability. By Lemma 1, we know that the following domain $D$ is invariable:

$$
D=\left\{(x, y) \mid 0 \leq x \leq \frac{2 \sigma}{\delta}, 0 \leq y \leq \frac{2}{\beta}\right\} .
$$

Then it is easy to prove that $d x / d t<0$ as $x>2 \sigma / \delta$. In addition, when $y_{0}>2 / \beta$,

$$
\frac{d y}{d t}=f y(1-\beta y-x)=-f y((1-\beta y)+x y)<0 .
$$

This shows that the vector fields are moving towards $D$ as $t$ increases.

Let Dulac function $B=1 / y$. Then

$$
\frac{\partial(B P)}{\partial x}+\frac{\partial(B Q)}{\partial y} \leq \alpha-\mu-f \beta<0 .
$$

Hence there are no closed trajectory surrounds $P_{0}$ in field $D$. That is, the result follows.

Using the original parameters, we can give the results as follows.
Theorem 4. If $s>d a / n, d b>m>p / g$, system (5) has only one critical equilibrium $P_{0}=(\sigma / \delta, 0)$. Furthermore, $P_{0}$ is globally asymptotically stable.

Remark 5. Theorem 4 is an instructive results to kill the tumor cells. The tumor cells will be killed out by the immune cells sooner or later under the above conditions; then we only need to take the necessary measures to control the parameters to satisfy the inequity in Theorem 4 .

In the following, we will study the existence and stability of the tumor-present equilibrium $P_{1}\left(x^{*}, y^{*}\right)$. Similar to the proof of Lemma 2.4 in [12], we can easily obtain the following results.

Theorem 6. For the number of positive equilibria, we can get the following results:

(1) If $>\delta>\mu / \beta>\mu, \sigma<f \delta$, and $\Delta<0$, then (5) has three distinct positive roots.

(2) If one of the following conditions is satisfied, then (5) has two distinct positive roots:

(a) $\alpha>\delta>\mu / \beta>\mu, \sigma>f \delta$, and $\Delta<0$.

(b) $\alpha>\delta>\mu / \beta>\mu, \sigma>f \delta$ and $\Delta=0$.

(3) Assume one of the following conditions is satisfied, then (5) has one positive root:

(a) $\alpha>\delta>\mu / \beta>\mu, \sigma<f \delta$, and $\Delta<0$.

(b) $\alpha>\delta>\mu / \beta>\mu, \sigma<f \delta$, and $A=B=0$.

(c) $\delta>\alpha, \beta>1, \alpha+\beta \delta<\mu, \sigma<\delta f$, and $\Delta>0$.

(d) $\alpha>\delta>\mu / \beta>\mu, \sigma<f \delta$, and $\Delta=0$.

The corresponding Jacobian matrix at $P_{1}\left(x^{*}, y^{*}\right)$ is

$$
\mathbf{J}=\left(\begin{array}{cc}
\frac{-\sigma}{x^{*}} & \frac{\alpha x^{*}}{\left(1+y^{*}\right)^{2}}-\mu x^{*} \\
-y^{*} & -f \beta y^{*}
\end{array}\right)
$$

Thus, we can give the following results.

Theorem 7. If $\sigma \beta>\mu f$, then $P_{1}$ is stable. And no Hopf bifurcation appears around the equilibrium $P_{1}$.

Proof. It is easy to see $\operatorname{Tr}\left(J\left(P_{1}\right)\right)=-\left(\sigma / x^{*}+f \beta y^{*}\right)<0$. Noting $\sigma \beta>\mu f$, it is easy to see that

$$
\begin{aligned}
\operatorname{det}\left(J\left(P_{1}\right)\right) & \geq \frac{y^{*} x^{*}}{\left(1+y^{*}\right)^{2}}\left[\left(\frac{\sigma \beta}{f}-\mu\right)\left(1+y^{*}\right)^{2}+\alpha\right] \\
& >0 .
\end{aligned}
$$

Thus the results are proved.

Theorem 8. Let $\left(x^{*}, y^{*}\right)$ be the coordinate of the positive equilibrium $P_{1}$. Then the following results hold:

(1) If $f \sigma \beta \geq \mu x^{* 2}$, then $P_{1}$ is stable. 
(2) If $f \sigma \beta<\mu x^{* 2}$, then $P_{1}$ is stable for $y^{*}>$ $\sqrt{\alpha x^{* 2} /\left(\mu x^{* 2}-f \sigma \beta\right)}-1$ and unstable for $0<y^{*}<$ $\sqrt{\alpha x^{* 2} /\left(\mu x^{* 2}-f \sigma \beta\right)}-1$.

Proof. It is easy to see

$$
\operatorname{det}\left(J\left(P_{1}\right)\right)=\frac{y^{*} x^{*}}{\left(1+y^{*}\right)^{2}}\left[a y^{* 2}+2 a y^{*}+a+\alpha\right],
$$

where $a=1 / x^{* 2}\left(\sigma f \beta-\mu x^{* 2}\right)$.

Let

$$
h\left(y^{*}\right)=a y^{* 2}+2 a y^{*}+a+\alpha .
$$

Then the sign of the $\operatorname{det}\left(J\left(P_{1}\right)\right)$ is the same as that of $h\left(y^{*}\right)$. Let $\Delta=4 \alpha\left(\mu-\sigma f \beta / x^{* 2}\right)$. If $f \sigma \beta=\mu x^{* 2}$ for any $y^{*}>0$, then $a=\Delta=0$. Hence,

$$
h\left(y^{*}\right)=a y^{* 2}+2 a y^{*}+a+\alpha=\alpha>0 .
$$

Then the first result can be obtained easily.

If $f \sigma \beta>\mu x^{* 2}$, that is, $a>0, \Delta<0$, then $h\left(y^{*}\right)>0$ for any $y^{*}>0$. That is to say, $P_{1}$ is stable as $f \sigma \beta>\mu x^{* 2}$. On the other hand, one knows $a<0$ as $f \sigma \beta<\mu x^{* 2}$. Noting $\alpha>\mu$, then

$$
a+\alpha=\frac{1}{x^{* 2}}\left(\sigma f \beta-\mu x^{* 2}+\alpha x^{* 2}\right)>0
$$

Then $h\left(y^{*}\right)=0$ has roots $-\sqrt{\alpha x^{* 2} /\left(\mu x^{* 2}-f \sigma \beta\right)}-1<0$ and $\sqrt{\alpha x^{* 2} /\left(\mu x^{* 2}-f \sigma \beta\right)}-1>0$. From $y^{*}>0$, we have $h\left(y^{*}\right)<0$ as $y^{*}>\sqrt{\alpha x^{* 2} /\left(\mu x^{* 2}-f \sigma \beta\right)}-1$ and $h\left(y^{*}\right)>0$ as $0<y^{*}<\sqrt{\alpha x^{* 2} /\left(\mu x^{* 2}-f \sigma \beta\right)}-1$. That is to say, the second result holds.

In the following, we give some simulation results of the above results. We consider the system (5) and the parameters suggested by V. A. Kuznetsov et al. Then system (5) becomes

$$
\begin{aligned}
\frac{d x}{d t}= & 0.1181+\frac{1.131 x(t) y(t)}{1+y(t)}-0.00311 x(t) y(t) \\
& -0.3743 x(t), \\
\frac{d y}{d t}= & 1.636 y(t)(1-0.002 y(t))-x(t) y(t) .
\end{aligned}
$$

Obviously, system (24) has three positive equilibria: $E_{1}(1.6348,0.366269), E_{2}(0.6538,300.188)$, and $E_{3}(0.1906$, 441.757). By simple computation, it is easy to know that the eigenvalues of $E_{1}$ are $-0.0367189 \pm 0.599721 i$, and $E_{2}$ has eigenvalues -1.45811 and 0.295245 , and $E_{3}$ has eigenvalues -1.68973 and -0.375447 . These results are represented in Figures 1 and 2.

\section{Dynamical Behaviors of the Model with Delay}

In this section, we shall consider model (5) with delay

$$
\begin{aligned}
& \frac{d x}{d t}=\sigma+\frac{\alpha x y}{1+y}-\mu x(t-\tau) y(t-\tau)-\delta x, \\
& \frac{d y}{d t}=f y(1-\beta y)-x y .
\end{aligned}
$$

The existence of the equilibria is the same as those of (5). The dynamical behaviors of the trivial equilibria and semitrivial equilibria are not difficult; we only study the positive equilibrium $P_{1}=\left(x^{*}, y^{*}\right)$ here. The characteristic equation of linearized system for (25) at $P_{1}$ takes the following form:

$$
\lambda^{2}+B \lambda+C+(D \lambda+E) e^{-\lambda \tau}=0,
$$

where

$$
\begin{aligned}
B & =f \beta y^{*}-\frac{\alpha y^{*}}{1+y^{*}}+\delta \\
& =\frac{f \beta y^{*}+f \beta y^{* 2}+\delta+y^{*}(\delta-\alpha)}{1+y^{*}}, \\
C & =\frac{f \beta y^{* 2}(\delta-\alpha)+f \beta y^{*}(2 \delta-\alpha)+f \beta \delta+\alpha x^{*}}{\left(1+y^{*}\right)^{2}}, \\
D & =\mu y^{*}, \\
E & =\mu y^{* 2} f \beta-\mu x^{*} y^{*}=\mu f y^{*}\left(2 \beta y^{*}-1\right) .
\end{aligned}
$$

Similar to the proof in [7], then the following results can be obtained easily.

Theorem 9. (1) If

$$
\begin{aligned}
B+D & >0, \\
C+E & >0, \\
D^{2}-B^{2}+2 C & <0, \\
C^{2}-E^{2} & >0 \text { or }\left(D^{2}-B^{2}+2 C\right)^{2}<4\left(C^{2}-E^{2}\right),
\end{aligned}
$$

then all roots of (26) have negative real parts for all $\tau \geq 0$.

(2) If $C^{2}-E^{2}<0$ or $D^{2}-B^{2}+2 C>0$ and $\left(D^{2}-B^{2}+2 C\right)^{2}=$ $4\left(C^{2}-E^{2}\right)$, then (26) has a pair of purely imaginary roots $\pm i \omega_{+}$ at $\tau=\tau_{j}^{+}$.

(3) If $D^{2}-B^{2}+2 C>0, C^{2}-E^{2}>0$ and $\left(D^{2}-B^{2}+\right.$ $2 C)^{2}>4\left(C^{2}-E^{2}\right)$, then (26) has a pair of purely imaginary roots $\pm i \omega_{+}\left( \pm i \omega_{-}\right.$, resp. $)$at $\tau=\tau_{j}^{+}\left(\tau=\tau_{j}^{-}\right.$,resp. $)$, where

$$
\omega^{2} \pm=\frac{1}{2}\left[\left(D^{2}-B^{2}+2 C\right) \pm \sqrt{\left(D^{2}-B^{2}+2 C\right)^{2}-4\left(C^{2}-E^{2}\right)}\right],
$$




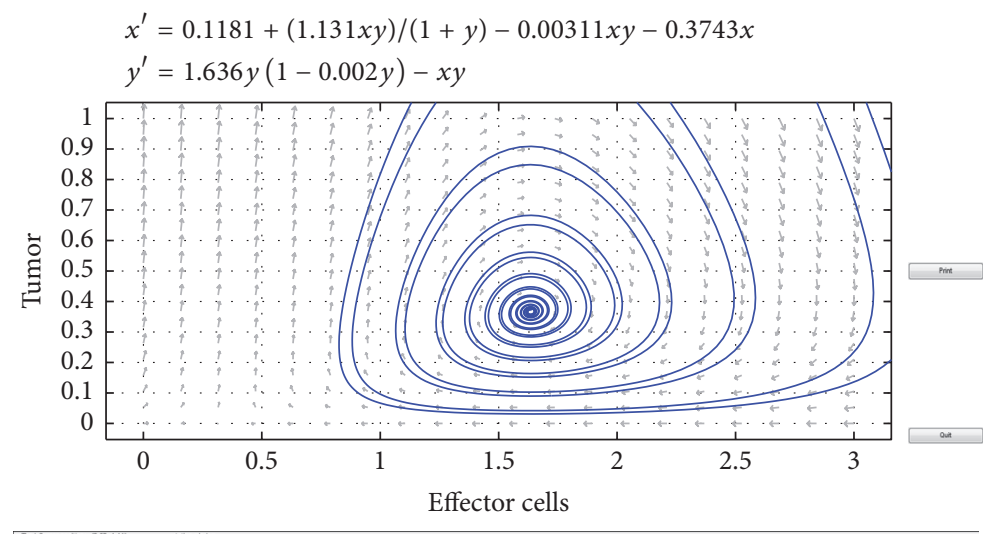

(a)

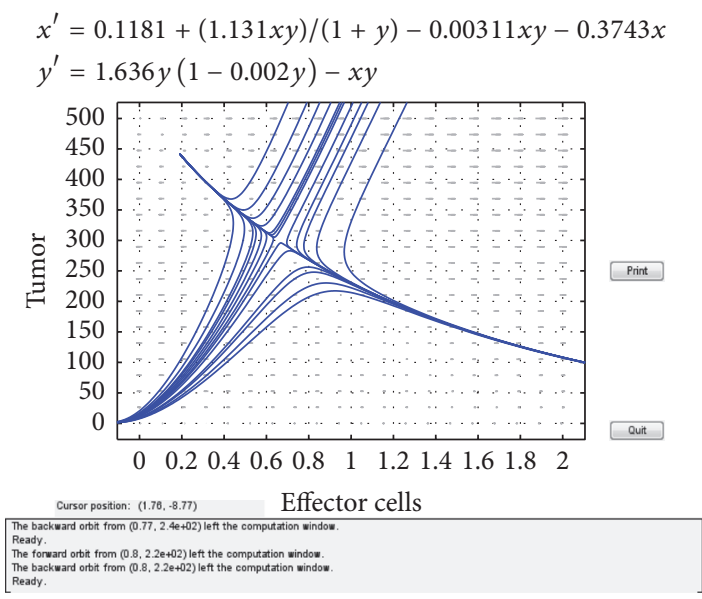

(b)

FIgURE 1: (a) Stable equilibrium $E_{1}$. (b) Saddle-node equilibrium $E_{2}$.

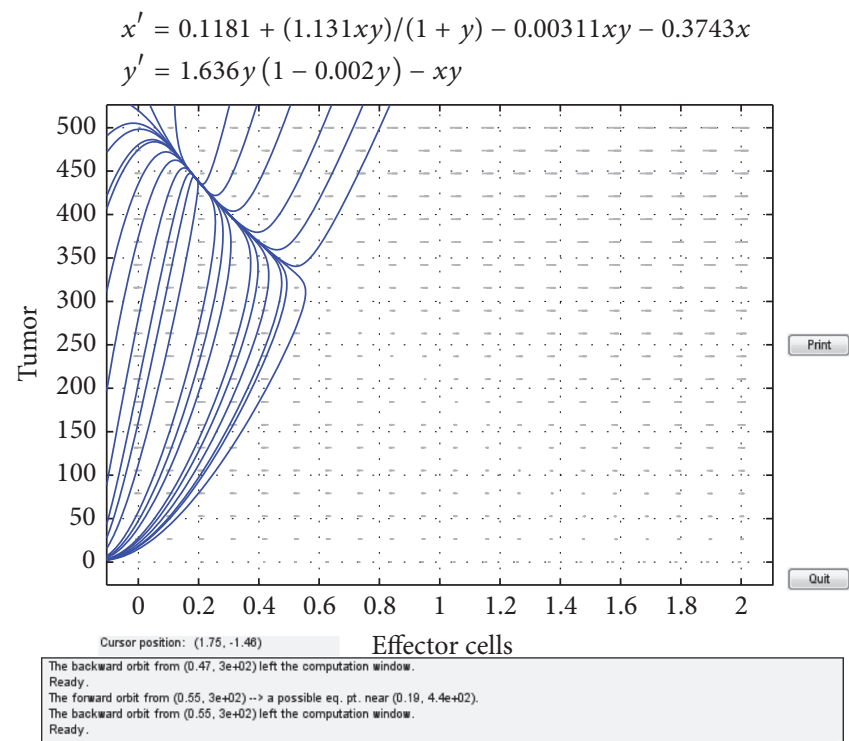

(a)

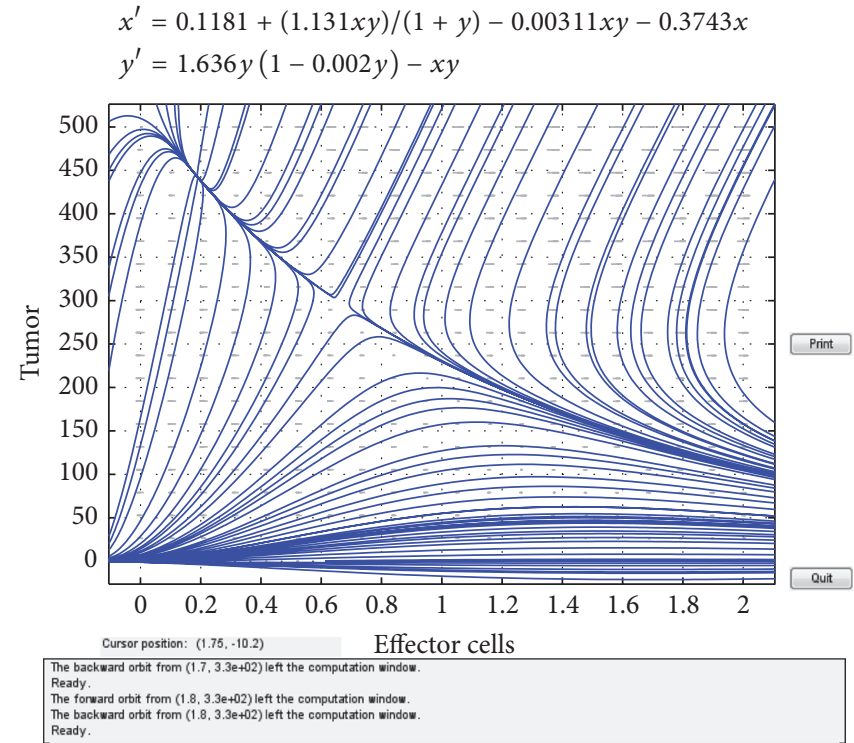

(b)

Figure 2: (a) Stable focus equilibrium $E_{3}$. (b) Saddle node $\left(E_{2}\right)$ and stable focus $\left(E_{3}\right)$.

$$
\tau_{j}^{ \pm}= \begin{cases}\frac{1}{\omega_{ \pm}}\left(2 j \pi+\arccos \frac{\left(\omega^{2}-C\right) E-D B \omega^{2}}{E^{2}+D^{2} \omega^{2}}\right), & D \omega^{2}-C D+B E \geq 0, j=1,2, \ldots \\ \frac{1}{\omega_{ \pm}}\left((2 i+1) \pi-\arccos \frac{\left(\omega^{2}-C\right) E-D B \omega^{2}}{E^{2}+D^{2} \omega^{2}}\right), & D \omega^{2}-C D+B E<0, i=1,2, \ldots\end{cases}
$$

Theorem 10. (1) If the conditions of (1) in Theorem 9 hold, then $P_{1}$ is asymptotically stable for any $\tau>0$.

(2) If $C^{2}<E^{2}$ or the conditions of (3) in Theorem 9 hold, then $E^{*}$ undergoes Hopf bifurcation as $\tau=\tau_{0}$.

(3) If $D^{2}-B^{2}+2 C>0,\left(D^{2}-B^{2}+2 C\right)^{2}=4\left(C^{2}-E^{2}\right)$ and $B^{2}+2 D \tau E+\tau^{2}>D^{2}+E^{2}$, then $E^{*}$ undergoes a Hopf bifurcation as $\tau=\tau_{0}$.
Proof. From the analysis of the above, we only need to compute

$$
\begin{aligned}
& \left.\operatorname{Sign} \frac{d(\operatorname{Re} \lambda)}{d \tau}\right|_{\lambda=i \omega} \\
& =\operatorname{Sign} \pm \sqrt{\left(D^{2}-B^{2}+2 C\right)^{2}-4\left(C^{2}-E^{2}\right)}
\end{aligned}
$$


In third case, $d(\operatorname{Re} \lambda) /\left.d \tau\right|_{\tau=\tau_{0}}=0$, then we need the sign of the second derivative of $\operatorname{Re} \lambda(\tau)$ of $(26)$ at the point where We can easily obtain $\lambda$ is passing through $\lambda_{0}$.

$$
\left.\operatorname{Sign}\left(\operatorname{Re} \lambda^{\prime \prime}\right)\right|_{\lambda=i \omega}=\frac{\omega^{2}\left(E^{2}+D^{2} \omega^{2}\right)^{2}\left(E^{2}+D^{2} \omega^{2}\left(2 \omega^{2}-2 C-2 D \tau E-\tau^{2}\right)-2 E^{2} A-A^{2}\right)}{A^{3}}
$$

with

$$
A=-B \omega^{2}-B C-D E+\tau \omega\left(E^{2}+D^{2} \omega^{2}\right) .
$$

Since sgn $\left.\operatorname{Re}(d \lambda / d \tau)^{-1}\right|_{\lambda=i \omega}=0,\left.\operatorname{sgn} \operatorname{Im}(d \lambda / d \tau)^{-1}\right|_{\lambda=i \omega} \neq$ 0 . Thus it is easy to see that $E^{2}+D^{2} \omega^{2}\left(2 \omega^{2}-2 C-2 D \tau E-\right.$ $\left.\tau^{2}\right)-2 E^{2} A-A^{2}<0$. This finishes the proof.

In the above, we obtained the conditions under which a family of periodic solutions bifurcated from the positive equilibrium at $\tau=\tau_{0}$. In the following, we derive the explicit formulae for determining the properties of the Hopf bifurcated solution by using the normal form and the center manifold theory. Throughout this section, we always assume that the system (25) undergoes Hopf bifurcations at the positive equilibrium $P_{1}\left(x^{*}, y^{*}\right)$ for $\tau=\tau_{0}$ and $\pm i \omega$ are the corresponding pure imaginary roots.

Let $t=\tau t^{\prime}, x(t)=x\left(\tau t^{\prime}\right)$. Then (25) becomes

$$
\begin{aligned}
& \frac{d x}{d t}=\tau\left[\sigma+\frac{\alpha x y}{1+y}-\mu x(t-1) y(t-1)-\delta x\right], \\
& \frac{d y}{d t}=\tau(f y(1-\beta y)-x y) .
\end{aligned}
$$

Set $\tau=\tau_{0}+p, p \in R$. Then $p=0$ is a Hopf bifurcation value for (33). Equation (5) can be written as

$$
x^{\prime}(t)=L_{p}\left(x_{t}\right)+F\left(p, x_{t}\right)
$$

where $x(t)=\left(x_{1}(t), x_{2}(t)\right)^{T} \in R^{2}$, and $L_{p}: C \rightarrow R, F:$ $R \times C \rightarrow R$ are given, respectively, by

$$
\begin{aligned}
\mathbf{L}_{p}(\phi) & =A_{1}\left(\begin{array}{l}
\phi_{1}(0) \\
\phi_{2}(0)
\end{array}\right)+A_{2}\left(\begin{array}{l}
\phi_{1}(-1) \\
\phi_{2}(-1)
\end{array}\right) \\
\mathbf{F}(p, \phi) & =\left(\tau_{0}+p\right)\left(\begin{array}{c}
\frac{-\alpha x^{*}}{\left(1+y^{*}\right)^{3}}(\phi)_{2}^{2}(0)+\frac{\alpha}{2\left(1+y^{*}\right)^{2}} \phi_{1}(0) \phi_{2}(0)-\mu \phi_{1}(-1) \phi_{2}(-1) \\
-f \beta(\phi)_{2}^{2}(0)-\phi_{1}(0) \phi_{2}(0)
\end{array}\right),
\end{aligned}
$$

with $\phi(0)=\left(\phi_{1}(0), \phi_{2}(0)\right)^{T} \in C$ and

$$
\begin{aligned}
& A_{1}=\left(\tau_{0}+p\right)\left(\begin{array}{cc}
\frac{\alpha y^{*}}{1+y^{*}}-\delta & \frac{\alpha x^{*}}{\left(1+y^{*}\right)^{2}} \\
-y^{*} & -f \beta y^{*}
\end{array}\right), \\
& A_{2}=\left(\tau_{0}+p\right)\left(\begin{array}{cc}
-\mu y^{*} & -\mu x^{*} \\
0 & 0
\end{array}\right) .
\end{aligned}
$$

By Riesz representation theorem, there exists a function $\eta(\theta, p)$ of bounded variation for $\theta \in[-1,0]$ such that

$$
L_{p}(\theta)=\int_{-1}^{0} d \eta(\theta, p) \phi(\theta) .
$$

For $\phi \in C^{1}\left([-1,0], R^{3}\right)$, define

$$
A(p) \phi= \begin{cases}\frac{d \phi(\theta)}{d \theta}, & \theta \in[-1,0), \\ \int_{-1}^{0} d \eta(s, p) \phi(s), & \theta=0,\end{cases}
$$

$$
R(p) \phi= \begin{cases}0, & \theta \in[-1,0) \\ F(p, \phi), & \theta=0 .\end{cases}
$$

Then system (34) is equivalent to

$$
x_{t}^{\prime}=A(p)\left(x_{t}\right)+R(p) x_{t},
$$

where $x_{t}(\theta)=x(t+\theta)$ for $\theta \in[-1,0]$. For $\Psi \in C^{1}([0,1]$, $\left.\left(R^{3}\right)^{*}\right)$, define

$$
A^{*} \psi(s)= \begin{cases}\frac{d \psi(s)}{d s}, & s \in(0,1], \\ \int^{-} 1_{0} d \eta^{T}(t, 0) \psi(-t), & s=0,\end{cases}
$$

and a bilinear inner product

$$
\begin{aligned}
\langle\psi(s), \phi(\theta)\rangle= & \bar{\psi}(0) \phi(0) \\
& -\int_{-1}^{0} \int_{\xi=0}^{\theta} \bar{\psi}(\xi-\theta) d \eta(\theta) \phi(\xi) d \xi,
\end{aligned}
$$

where $\eta(\theta)=\eta(\theta, 0)$. Then $A(0)$ and $A^{*}$ are joint operators. 
Suppose that $q(\theta)=(1, \rho)^{T} e^{i \theta \omega_{0} \tau_{0}}$ is the eigenvector of $A(\theta)$ corresponding to $i \omega_{0} \tau_{0}$, with $\rho=-y^{*} /\left(f \beta y^{*}+i \omega_{0} \tau_{0}\right)$. Then $q^{*}(s)=D\left(1, \rho^{*}\right) e^{i s \omega_{0} \tau_{0}}$ is the eigenvector of $A^{*}$ with $\rho^{*}=\left(1 / y^{*}\right)\left[\alpha y^{*} /\left(1+y^{*}\right)-\delta-\mu y^{*} e^{i \omega_{0} \tau_{0}}-i \omega_{0} \tau_{0}\right], D=$ $1 /\left(1+\bar{\rho} \rho^{*}+\tau\left(-\mu y^{*}-\mu x^{*} \bar{\rho}\right) e^{-i \omega_{0} \tau_{0}}\right)$ such that $\left\langle q^{*}(s), q(\theta)\right\rangle=1$, $\left\langle q^{*}(s), \bar{q}(\theta)\right\rangle=0$.

In the following, we use the ideas in Adam and Bellomo [1] to compute the coordinates describing center manifold $C_{0}$ at $p=0$. Define

$$
\begin{aligned}
z(t) & =\left\langle q^{*}, x_{t}\right\rangle, \\
W(t, \theta) & =x_{t}(\theta)-2 \operatorname{Re} z(t) q(\theta) .
\end{aligned}
$$

On the center manifold $C_{0}$, we have

$$
\begin{aligned}
W(t, \theta)= & W(Z(t), \bar{z}(t), \theta) \\
= & W_{20}(\theta) \frac{z^{2}}{2}+W_{11}(\theta) z \bar{z}+W_{02}(\theta) \frac{\bar{z}^{2}}{2} \\
& +\cdots,
\end{aligned}
$$

where $z$ and $\bar{z}$ are local coordinates for the center manifold $C_{0}$ in the direction of $q^{*}$ and $\bar{q}^{*}$. Note that $W$ is real if $x_{t}$ is real. For $x_{t} \in C_{0}$ of (39), we have

$$
\begin{aligned}
z(t) & =i \omega_{0} \tau_{0} z+\bar{q}^{*}(0) F(0, W(z, \bar{z}+2 \operatorname{Re} z q(\theta))) \\
& =i \omega_{0} \tau_{0} z+g(z, \bar{z}),
\end{aligned}
$$

where

$$
\begin{aligned}
g(z, \bar{z})= & \bar{q}^{*}(0) F_{0}(Z(t), \bar{z}(t)) \\
= & g_{20}(\theta) \frac{z^{2}}{2}+g_{11}(\theta) z \bar{z}+g_{02}(\theta) \frac{\bar{z}^{2}}{2} \\
& +g_{21}(\theta) \frac{z^{2} \bar{z}}{2}+\cdots .
\end{aligned}
$$

From (43) and (44), we have

$$
x_{t}=\left(x_{1 t}(\theta), x_{2 t}(\theta)\right)=W(t, \theta)+z q(\theta)+\overline{z q(\theta)}
$$

and $q(\theta)=(1, \rho)^{T} e^{i \theta \omega_{0} \tau_{0}}$. Comparing the coefficients of the above equality with (45), we obtain

$$
\begin{aligned}
g_{20} & =2 \tau \bar{D}\left[\frac{\alpha x^{*}}{\left(1+y^{*}\right)^{3}} \rho^{2}+\frac{\alpha}{2\left(1+y^{*}\right)^{2}} \rho-\mu \rho e^{-2 i \omega_{0} \tau_{0}}\right. \\
& \left.-f \beta \rho^{2}-\rho\right], \\
g_{11} & =2 \tau \bar{D}\left[\frac{\alpha x^{*}}{\left(1+y^{*}\right)^{3}} \rho \bar{\rho}+\frac{\alpha}{2\left(1+y^{*}\right)^{2}} 2 \operatorname{Re} \rho\right. \\
& -\mu 2 \operatorname{Re} \rho-f \beta \rho \bar{\rho}-2 \operatorname{Re} \rho], \\
g_{02} & =2 \tau \bar{D}\left[\frac{\alpha x^{*}}{\left(1+y^{*}\right)^{3}} \bar{\rho}^{2}+\frac{\alpha}{2\left(1+y^{*}\right)^{2}} \bar{\rho}-\mu \bar{\rho} e^{2 i \omega_{0} \tau_{0}}\right.
\end{aligned}
$$

$$
\begin{aligned}
& \left.-f \beta \bar{\rho}^{2}-\bar{\rho}\right]+\frac{\alpha x^{*}}{\left(1+y^{*}\right)^{3}} W_{11}^{2}(0) \rho \\
+ & z^{2} \bar{z}\left[\left(\frac { \alpha } { 2 ( 1 + y ^ { * } ) ^ { 2 } } \left(W_{20}^{1}(0) \bar{\rho}+W_{20}^{2}(0)\right.\right.\right. \\
& \left.\left.\left.+W_{11}^{1}(0) \rho+W_{11}^{2}(0)\right)+\frac{\alpha x^{*}}{\left(1+y^{*}\right)^{3}} W_{20}^{2}(0) \bar{\rho}\right)\right], \\
g_{21} & =2 \tau \bar{D}\left[\frac{\alpha x^{*}\left(W_{20}^{2}(0) \bar{\rho}+W_{11}^{2}(0) \rho\right)}{\left(1+y^{*}\right)^{3}}\right. \\
& +\frac{\alpha\left(W_{20}^{1}(0) \bar{\rho}+W_{20}^{2}(0)+W_{11}^{1}(0) \rho+W_{11}^{2}(0)\right)}{2\left(1+y^{*}\right)^{2}} \\
& +\mu\left(W_{20}^{1}(-1) \rho e^{-2 i \omega_{0} \tau_{0}}+W_{20}^{2}(-1) e^{-2 i \omega_{0} \tau_{0}}\right. \\
& \left.+W_{11}^{1}(-1) \rho e^{-2 i \omega_{0} \tau_{0}}+W_{11}^{2}(-1) e^{-2 i \omega_{0} \tau_{0}}\right) \\
& -f \beta\left(W_{20}^{2}(0) \bar{\rho}+W_{11}^{2}(0) \rho\right)-\left(W_{20}^{1}(0) \bar{\rho}\right. \\
& \left.\left.+W_{20}^{2}(0)+W_{11}^{1}(0) \rho+W_{11}^{2}(0)\right)\right] .
\end{aligned}
$$

In order to determine $g_{21}$, we also need to compute $W_{20}(\theta)$ and $W_{11}(\theta)$ as follows:

$$
\begin{aligned}
\dot{W} & =\dot{x}_{t}-\dot{z} q-\dot{\overline{z q}} \\
& = \begin{cases}A W-2 \operatorname{Re} \overline{q^{*}}(0) F_{0} q(\theta), & \theta \in[-1,0), \\
A W-2 \operatorname{Re} \overline{q^{*}}(0) F_{0} q(\theta)+F_{0}, & \theta=0,\end{cases} \\
& =A W+H(z, \bar{z}, \theta),
\end{aligned}
$$

where

$$
H(z, \bar{z}, \theta)=H_{20}(\theta) \frac{z^{2}}{2}+H_{11}(\theta) z \bar{z}+H_{02}(\theta) \frac{\bar{z}^{2}}{2}
$$

On the center manifold $C_{0}$ near the origin,

$$
\dot{W}=W_{z} \dot{z}+W_{\bar{z}} \dot{\bar{z}} .
$$

Comparing the coefficients of $z$, we obtain

$$
\begin{aligned}
(A-2 i \omega \tau) W_{20}(\theta) & =-H_{20}(\theta), \\
A W_{11} & =-H_{11}(\theta), \\
H_{20}(\theta) & =-g_{20} q(\theta)-\bar{g}_{02} \bar{\theta}, \\
H_{11}(\theta) & =-g_{11} q(\theta)-\bar{g}_{11} \bar{q}(\theta) .
\end{aligned}
$$

From (52) and the definition of $A$, one has

$$
\begin{aligned}
\dot{W}_{20}(\theta)= & 2 i \omega_{0} \tau_{0} W_{20}(\theta)+g_{20} q(0) e^{i \omega_{0} \tau_{0} \theta} \\
& +\bar{g}_{02} \bar{q}(0) e^{-i \omega_{0} \tau_{0} \theta}
\end{aligned}
$$


Solving the above ODE, then

$$
\begin{aligned}
W_{20}(\theta)= & -\frac{i g_{20}}{\omega_{0} \tau_{0}} q(0) e^{i \omega_{0} \tau_{0} \theta}-\frac{i \bar{g}_{02}}{3 \omega_{0} \tau_{0}} \bar{q}(0) e^{-i \omega_{0} \tau_{0} \theta} \\
& +E_{1} e^{2 i \omega_{0} \tau_{0} \theta}
\end{aligned}
$$

where $E_{1}=\left(E_{1}^{(1)}, E_{1}^{(2)}\right) \in R^{2}$ is a constant vector. Similarly, we obtain

$$
\dot{W}_{11}=\frac{i g_{11}}{\omega_{0} \tau_{0}} q(0) e^{i \omega_{0} \tau_{0} \theta}-\frac{i \bar{g}_{11}}{\omega_{0} \tau_{0}} \bar{q}(0) e^{-i \omega_{0} \tau_{0} \theta}+E_{2},
$$

where $E_{2}=\left(E_{2}^{(1)}, E_{2}^{(2)}\right) \in R^{2}$ is a constant vector.

From the definition of $A$ and the above equations, we can obtain

$$
=2 A\left(\begin{array}{c}
\frac{\alpha x^{*}}{\left(1+y^{*}\right)^{3}} \rho^{2}+\frac{\alpha}{2\left(1+y^{*}\right)^{2}} \rho-\mu \rho e^{-2 i \omega_{0} \tau_{0}} \\
0 \\
-f \beta \bar{\rho}^{2}-\bar{\rho}
\end{array}\right),
$$

with

$$
=\left(\begin{array}{cc}
2 i \omega_{0}+\frac{\alpha y^{*}}{1+y^{*}}-d-\mu y^{*} e^{2 i \omega_{0} \tau_{0}} & \frac{\alpha x^{*}}{\left(1+y^{*}\right)^{2}}-\mu x e^{2 i \omega_{0} \tau_{0}} \\
-y^{*} & 2 i \omega_{0}-f \beta y^{*}
\end{array}\right)^{-1} .
$$

In the same way, it is easy to get

$$
\begin{aligned}
& E_{2}=2\left(\begin{array}{cc}
\frac{\alpha y^{*}}{1+y^{*}}-d-\mu y^{*} & \frac{\alpha x^{*}}{\left(1+y^{*}\right)^{2}}-\mu x \\
-y^{*} & 2 i \omega_{0}-f \beta y^{*}
\end{array}\right)^{-1} \\
& \left(\begin{array}{c}
\frac{\alpha x^{*}}{\left(1+y^{*}\right)^{3}} \rho \bar{\rho}+\frac{\alpha}{2\left(1+y^{*}\right)^{2}} 2 \operatorname{Re} \rho-\mu 2 \operatorname{Re} \rho \\
f \beta \bar{\rho}^{2}-\bar{\rho}
\end{array}\right) .
\end{aligned}
$$

In order to determine $E_{1}$ and $E_{2}$, we can calculate $W_{20}(\theta)$ and $W_{11}(\theta)$ from (54) and (55). Furthermore, we can also determine $g_{21}$. Thus we can be able to compute the following values:

$$
\begin{aligned}
c_{1}(0) & =\frac{i}{2 \omega_{0} \tau_{0}}\left(g_{20} g_{11}-2\left|g_{11}\right|^{2}-\frac{\left|g_{02}\right|^{2}}{3}\right)+\frac{g_{21}}{2}, \\
\mu_{2} & =-\frac{\operatorname{Rec} c_{1}(0)}{\operatorname{Re} \lambda^{\prime}\left(\tau_{0}\right)}, \\
\beta_{2} & =2 \operatorname{Rec} c_{1}(0), \\
T_{2} & =-\frac{\operatorname{Im} c_{1}(0)+\mu_{2} \operatorname{Im} \lambda^{\prime}\left(\tau_{0}\right)}{\omega_{0} \tau_{0}} .
\end{aligned}
$$

Theorem 11. Equation (25) undergoes Hopf bifurcation as $\tau=\tau_{0}$. If $\mu_{2}<0\left(\mu_{2}>0\right)$, then the bifurcated periodic solution is supercritical (subcritical); if $\beta<0(\beta<0)$, then the bifurcating solution is stable (unstable). The period of the bifurcated periodic solution is $T_{2}$.
In the following, we present some numerical results of system (25) at different values of $\tau$. We choose the parameters the same as before; the system (25) becomes

$$
\begin{aligned}
\frac{d x}{d t}= & 0.1181+\frac{1.131 x y}{1+y}-0.00311 x(t-\tau) y(t-\tau) \\
& -0.3743 x \\
\frac{d y}{d t}= & 1.636 y(1-0.002 y)-x y .
\end{aligned}
$$

Obviously, system (60) has three positive equilibria: $E_{1}(1.6348,0.366269), E_{2}(0.6538,300.188)$, and $E_{3}(0.1906$, 441.757). Equilibrium $E_{1}$ is stable for all $\tau$, while equilibrium $E_{2}$ is unstable saddle node for all $\tau$. When $\tau=0$, the positive equilibrium $E_{3}$ is a stable focus, as $\tau$ is growing to $\tau_{0}=0.707732, \lambda^{\prime}\left(\tau_{0}\right)=0.604929-0.176726 i$. When $\tau$ passes the critical value $\tau_{0}, E_{3}$ loses its stability and a Hopf bifurcation occurs; that is, a family of periodic solutions bifurcate from $E_{1}$. This is clearly demonstrated by Figure 3 .

\section{Conclusions}

In 1994, Kuznetsov et al. [11] introduced a model, which describes competition between the tumor and immune cells. This mode describes the response of effector cells to the growth of tumor cells. Later, the model is simplified to a two-dimensional differential equation with Lotka-Volterra form immune response function $F(E, T)=\theta E T$; the rich dynamical behaviors are studied such as stability, qualitative behaviors, and bifurcation behaviors.

In this paper, we studied the model with an immune response function with Michaelis-Menten form $F(E, T)=$ $p E T /(g+T)$. In this case, the properties of system (4) are more complicated. There are so many tedious computations even for the existence of the equilibria. The dynamical behaviors of the model are more rich.

We have studied the nonlinear dynamics of a twodimensional general differential system. We first provided linear analysis of the system at the possible equilibria, namely, the semitrivial and positive equilibria, and discussed the existence of Hopf bifurcation at the positive equilibrium. Then we consider the system with delay; we investigated the Hopf bifurcation of the system. The existence and stability of periodic solutions were given. Numerical simulations were presented to illustrate the theoretical analysis and results. Cancer immunosurveillance functions are taken as an important defense to cancer; this is the elimination process. In fact, the existence and stability of the semitrivial equilibrium correspond to the elimination process. Our results on the existence and stability of the Hopf bifurcated periodic solutions describe the equilibrium process. If a stable periodic orbit exists, then the tumor and the immune system can coexist for a long time, although the cancer cannot be eliminated. Furthermore, the parameters are important in controlling the development and progression of the tumor, which is decided by the conditions of the existence of the bifurcations. 


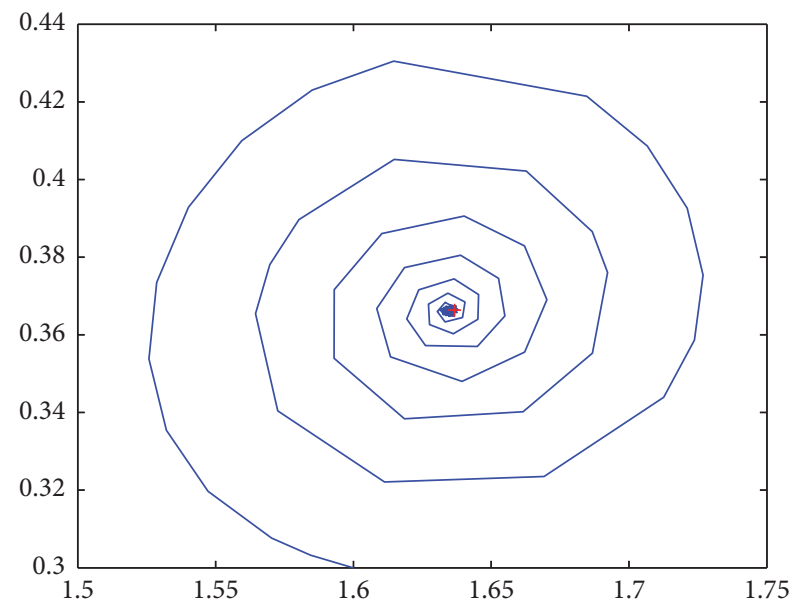

(a)

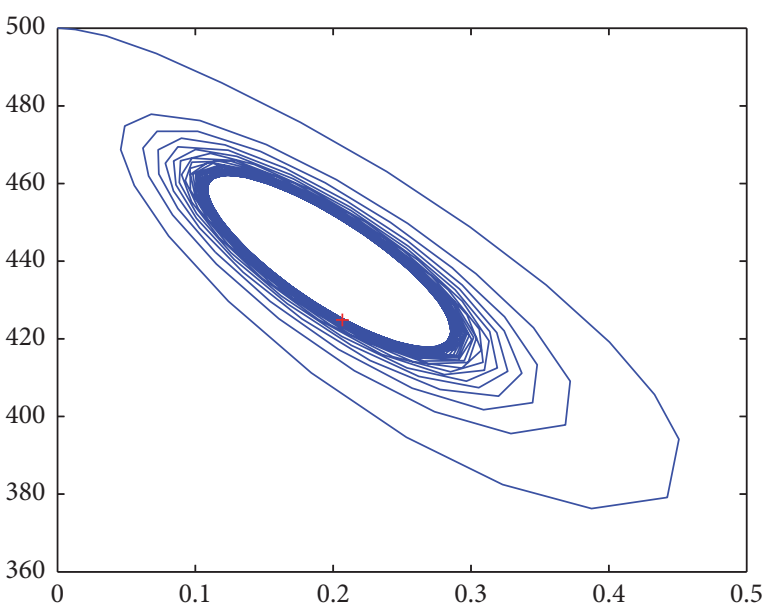

(b)

FIgURE 3: (a) Stable equilibrium $E_{1}$ as $\tau=1$. (b) Bifurcated solution around $E_{3}$ as $\tau=0.7$.

The existence of oscillatory solutions in the tummor and immune system interaction models demonstrates that the phenomenon has been observed in some related models [7, $10,13-15]$. The initial values and delay are also important in the oscillatory coexistence of the tumor cells and the effector cells. Numerical simulations indicate this information well.

\section{Conflicts of Interest}

The authors declare that there are no conflicts of interest regarding the publication of this paper.

\section{Acknowledgments}

This paper was supported by National Natural Science Foundation of China (11401217, 11671142, 11401060, and 11401117), Shanghai Leading Academic Discipline Project (B407), and 211 Project of Key Academic Discipline of East China Normal University.

\section{References}

[1] J. Adam and N. Bellomo, A Survey of Models on Tumor Immune Systems Dynamics, Birkhäuser, Boston, Mass, USA, 1996.

[2] A. R. A. Anderson, A. M. Weaver, P. T. Cummings, and V. Quaranta, "Tumor morphology and phenotypic evolution driven by selective pressure from the microenvironment," Cell, vol. 127, no. 5, pp. 905-915, 2006.

[3] J. C. Arciero, T. L. Jackson, and D. E. Kirschner, "A mathematical model of tumor-immune evasion and siRNA treatment," Discrete and Continuous Dynamical Systems-Series B, vol. 4, no. 1, pp. 39-58, 2004.

[4] M. Galach, "Dynamics of the tumor-immune system competition-the effect of time delay," International Journal of Applied Mathematics and Computer Science, vol. 13, no. 3, pp. 395-406, 2003.

[5] M. Owen and J. Sherratt, "Modeling the macrophage invasion of tumors: effects on growth and composition," Mathematical Medicine and Biology, vol. 15, pp. 165-185, 1998.
[6] L. G. de Pillis, A. E. Radunskaya, and C. L. Wiseman, "A validated mathematical model of cell-mediated immune response to tumor growth," Cancer Research, vol. 65, no. 17, pp. 79507958, 2005.

[7] P. Bi and H. Xiao, "Hopf bifurcation for tumor-immune competition systems with delay," Electronic Journal of Differential Equations, vol. 2014, no. 27, pp. 1-13, 2014.

[8] R. Yafia, "Hopf bifurcation in a delayed model for tumorimmune system competition with negative immune response," Discrete Dynamics in Nature and Society, vol. 2006, Article ID 95296, 9 pages, 2006.

[9] R. Yafia, "Hopf bifurcation analysis and numerical simulations in an ODE model of the immune system with positive immune response," Nonlinear Analysis: Real World Applications, vol. 8, no. 5, pp. 1359-1369, 2007.

[10] R. Yafia, "Hopf bifurcation in differential equations with delay for tumor-immune system competition model," SIAM Journal on Applied Mathematics, vol. 67, no. 6, pp. 1693-1703, 2007.

[11] V. A. Kuznetsov, I. A. Makalkin, M. A. Taylor, and A. S. Perelson, "Nonlinear dynamics of immunogenic tumors: parameter estimation and global bifurcation analysis," Bulletin of Mathematical Biology, vol. 56, no. 2, pp. 295-321, 1994.

[12] J. Li, Master degree dissertations [M.S. thesis], East China Normal University, 2014.

[13] P. Bi and S. Ruan, "Bifurcations in delay differential equations and applications to tumor and immune system interaction models," SIAM Journal on Applied Dynamical Systems, vol. 12, no. 4, pp. 1847-1888, 2013.

[14] P. Bi and H. Xiao, "Bifurcations of tumor-immune competition systems with delay," Abstract and Applied Analysis, vol. 2014, Article ID 723159, 12 pages, 2014.

[15] A. d'Onofrio and A. Gandolfi, "Tumour eradication by antiangiogenic therapy: analysis and extensions of the model by Hahnfeldt et al. (1999)," Mathematical Biosciences, vol. 191, no. 2, pp. 159-184, 2004. 


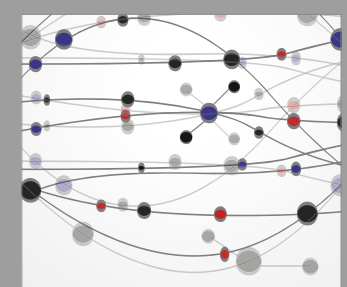

The Scientific World Journal
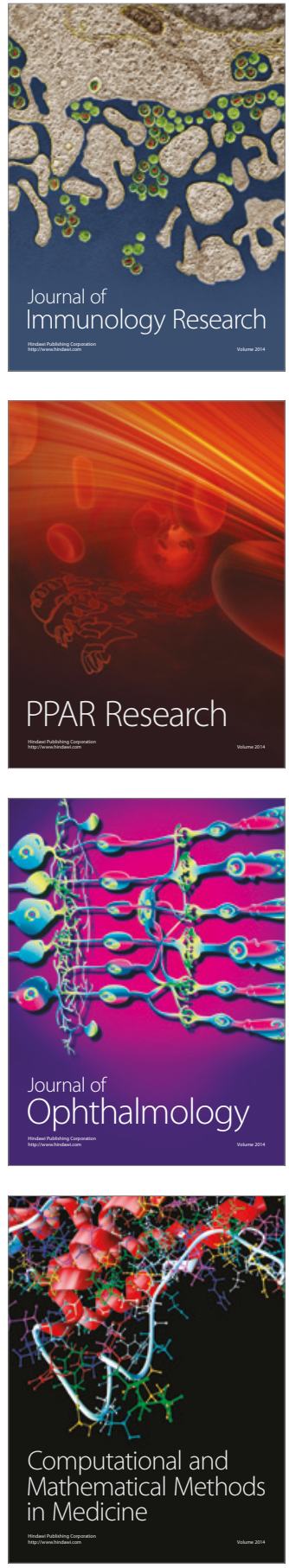

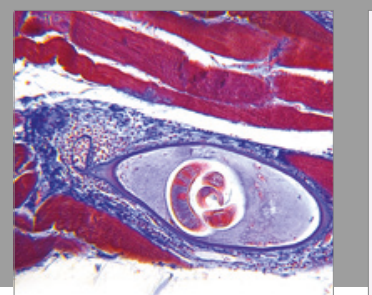

Gastroenterology Research and Practice
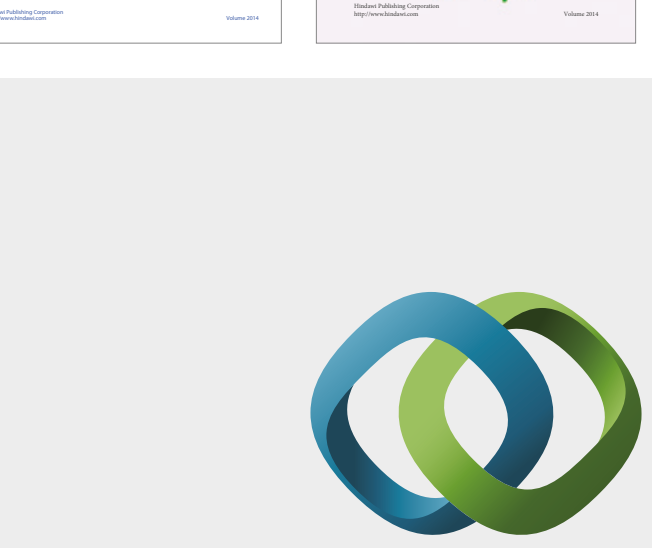

\section{Hindawi}

Submit your manuscripts at

https://www.hindawi.com
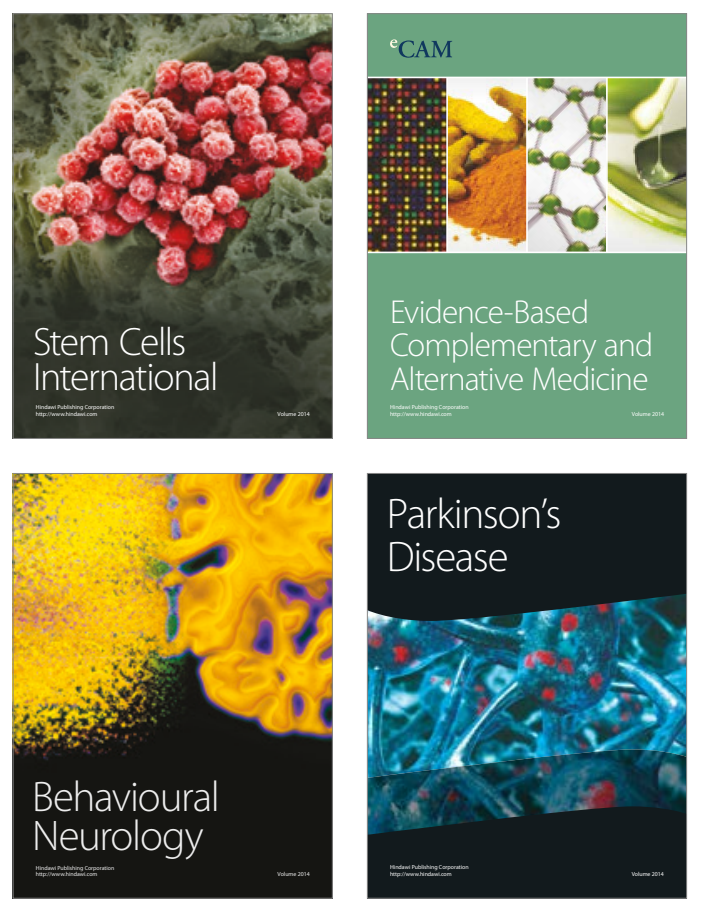
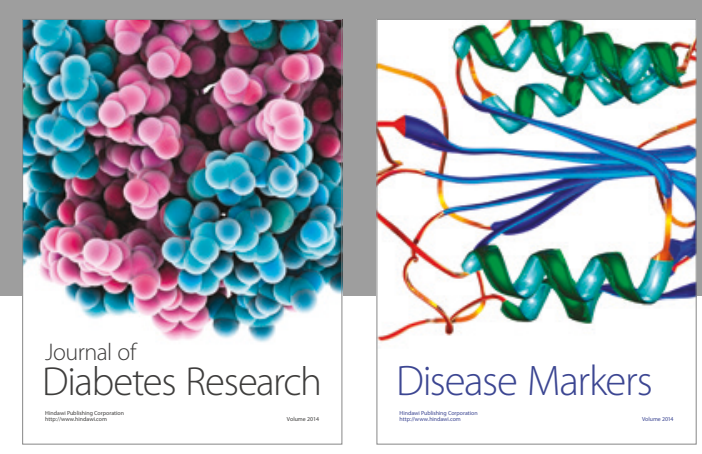

Disease Markers
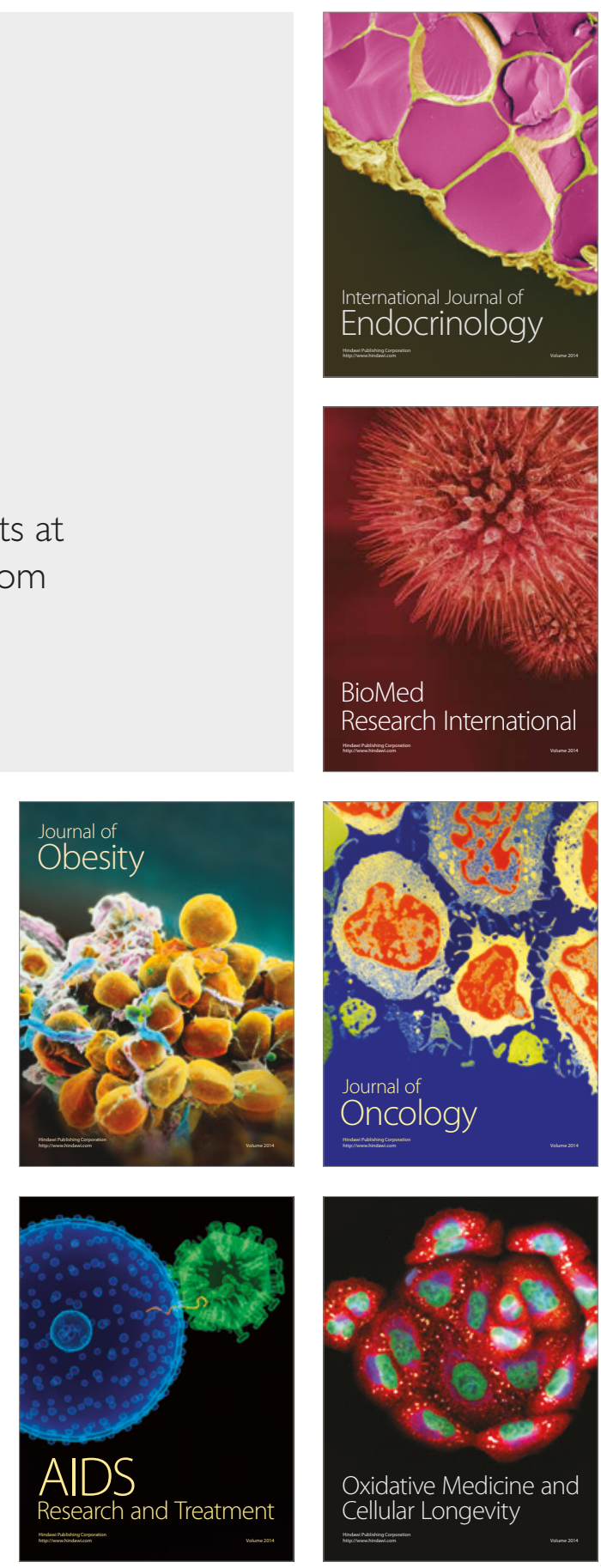